\title{
Oil Painting Creation Based on the Subject Matter of Miao Nationality Settlements in Guizhou
}

\author{
Xuemei Zhang ${ }^{1,2, *}$ \\ 1.Southwest Min Minzu University, Chengdu, Sichuan, China \\ 2.Aba Tea Teachers University, Aba, Sichuan, China \\ *snowzxm123@163.com
}

\begin{abstract}
In order to study the oil paintings of Miao nationality settlements in Guizhou, the development, changes and performance characteristics of the oil paintings of Miao nationality in Guizhou are systematically combed and summarized. Moreover, the influencing factors and ideological connotation of the oil paintings of Miao nationality in Guizhou are further analyzed. The results show that only when going deep into life and absorbing nutrients and selecting themes in life can good works that depict the people's spiritual outlook and show the spirit of the times be created. Thus, in the creation of oil paintings, it not only needs to reflect the progressive spirit of the times and lively social life in the performance of the subject matter, but also needs to develop in the oil painting performance language and other aspects.
\end{abstract}

Keywords: Guizhou; Miao nationality; subject matter; oil painting creation; artistic charm.

\section{Introduction}

Art is a kind of creation that transforms human ecology into intuitive aesthetic form. Everything in the original ecology is the most fundamental source of all artistic creation subject matters. With the development of human society, in all human spiritual production, art has a wealth of charm, and art creation is a mystery and difficult to understand [1]. The artistic charm of oil paintings of Miao nationality in Guizhou is taken as the research subject. The reason is that, in the development of modern and contemporary oil paintings, the creation of ethnic minority oil paintings is not only an indispensable part of the overall development of Chinese painting, but also an important factor in the composition of traditional Chinese culture, which occupies a very important position in the discipline construction of the arts and ethnology [2]. Guizhou's exquisite ethnic minority culture provides artists with abundant material for their art creation, but also shows its unique and most valuable humanistic value and the most charming artistic charm. Especially the colourful natural environment and strong ethnic customs in the areas inhabited by Miao nationality in Guizhou, it provides many different and unique original creation subject matters [3]. Oil paintings of Miao nationality in Guizhou grew up in this mysterious and ancient land. All its achievements and shortcomings, as well as brilliance and defects are derived from Guizhou's unique geography, history, politics and culture. It is these particularities that endow it with unique artistic character and enable it to become a dazzling pearl in Chinese oil paintings [4].

\section{State of the Art}

For the research on oil painting of Miao nationality in Guizhou, there is almost no record of relevant research trends abroad, and there is no relevant literature on it and no related research literature in foreign countries. Relatively speaking, there are still some research activities and literature achievements on ethnic minority oil painting creation in this subject field, but there is no systematic and targeted theoretical research on oil painting creation of Miao nationality in Guizhou. The Folklore of Chinese Ethnic Minorities, written by Mao Gongning and published by Ethnic Publishing House, was published in June 2006. According to its content and form, this book divides ethnic minority customs into 12 aspects, including clothing, diet, living, transportation, production, year-old festivals, life etiquette, social family, religious beliefs, taboos, art, and competitive entertainment. This provides a wide range of content for ethnic minority subject matters [5]. 
The book Creation of Ethnic Minority Oil Painting Themes written by Liu Wenbin and published by Hebei Fine Arts Publishing House was published in December 2009. This book is the latest theory on ethnic minority oil painting. The author of this book made a detailed analysis and discussion on the theme of Qinghai-Tibet Plateau, the theme of Silk Road in Northwest and Xinjiang, the theme of Inner Mongolia grassland, the theme of ethnic minorities in southwest China, and the case of painters. There is almost no mention of the painting of Miao nationality [6]. Nevertheless, Creation of Ethnic Minority Oil Painting Themes also provides a reliable theoretical basis for this topic.

\section{Methodology}

As an artist, life is creation, and creation is life. How to find and explore the intrinsic vitality and external charm of ethnic minority themes, from which to gain creative insights with the spirit of the times, is what our contemporary oil painting creation needs to think and explore. With the mystery and temptation of Miao village in Guizhou, the artists go deep into the areas where Miao people live together in Guizhou, feel, search and explore the national characteristics of oil painting creation in the strange life of ethnic minorities. The hardships they have tasted and the philosophies they have learned in the process of seeking folk customs and inspiring their creation should be a kind of real feeling experience in the world. This impulse of passion has brought great new and colourful works to the audience. These works, from different angles, show the historical and cultural heritage of ethnic minorities, the evolution of the modernization process, aesthetic taste and spiritual spirit.

In recent years, some painters have made reproducible art creations based on the natural environment and colourful national customs of the Miao nationality area in Guizhou Province. They depict the existence and state of the ancient Miao village in three-dimensional and multi-dimensional ways, showing the flowing history and long-standing spirit of the ancient Miao village, as well as describing and outlining its ancient and mysterious shadow. They can accurately express the national characteristics of oil painting of Miao nationality in Guizhou, and have high artistic value. They accurately and thoroughly reproduce their paintings, pouring into the sincerest and profound feelings of the Miao nationality in Guizhou. Now these oil paintings are sorted out and classified according to the theme of Miao nationality in Guizhou, which are mainly manifested in the natural environment, portraits, festivals, production and life scenes, wedding and funeral rites.

\section{Results and Discussion}

\subsection{Natural Environment Landscape}

The original natural environment is closely related to the life of the Miao nationality people. The description of the natural environment is naturally a common subject matter in oil painting. Artists who have always depicted ethnic minority themes will appreciate that the unique natural scenery in ethnic minority areas is particularly brilliant in oil painting creation compared with the high-rise buildings in cities and the artificial landscape. After experiencing the vivid natural scenery and combining his subjective consciousness and personal feelings, the artists are eager to express their inner feelings by using oil painting techniques. Therefore, the landscape oil painting which seeks artistic language in describing the natural scenery of the Miao nationality area in Guizhou wins the affirmation of the viewers, and forms the regional symbols of Guizhou, such as works of Chen Hongqi and Kong Yang.

Li Huiyong, born in June 1959 in Nangong, Hebei, now lives in Guiyang. In recent years, Li Huiyong has borrowed the stippling painting methods of Xiula and Colville, and integrated them into the artistic conception of Chinese classical aesthetics, creating a number of innovative local oil paintings. Among them, Li Huiyong spent a whole year on a painting of Spring Dyeing Miao Village, full of ethnic characteristics. Passing Through the Wind and Rain Bridge won the Silver Award for the third "West China's traditional Chinese painting and oil painting exhibition". The painting depicts a black ox walking on a dim wind-rain bridge. In front of it is a typical symbolic building, the drum tower, bathed in sunshine in the Miao village. The composition intentionally arranges a strong visual 
contrast between the dark and the bright in the picture, so that the whole work has a special effect like a printmaking, and the performance of this work is a useful exploration of the expression techniques of Guizhou local oil painting (Figure 1).

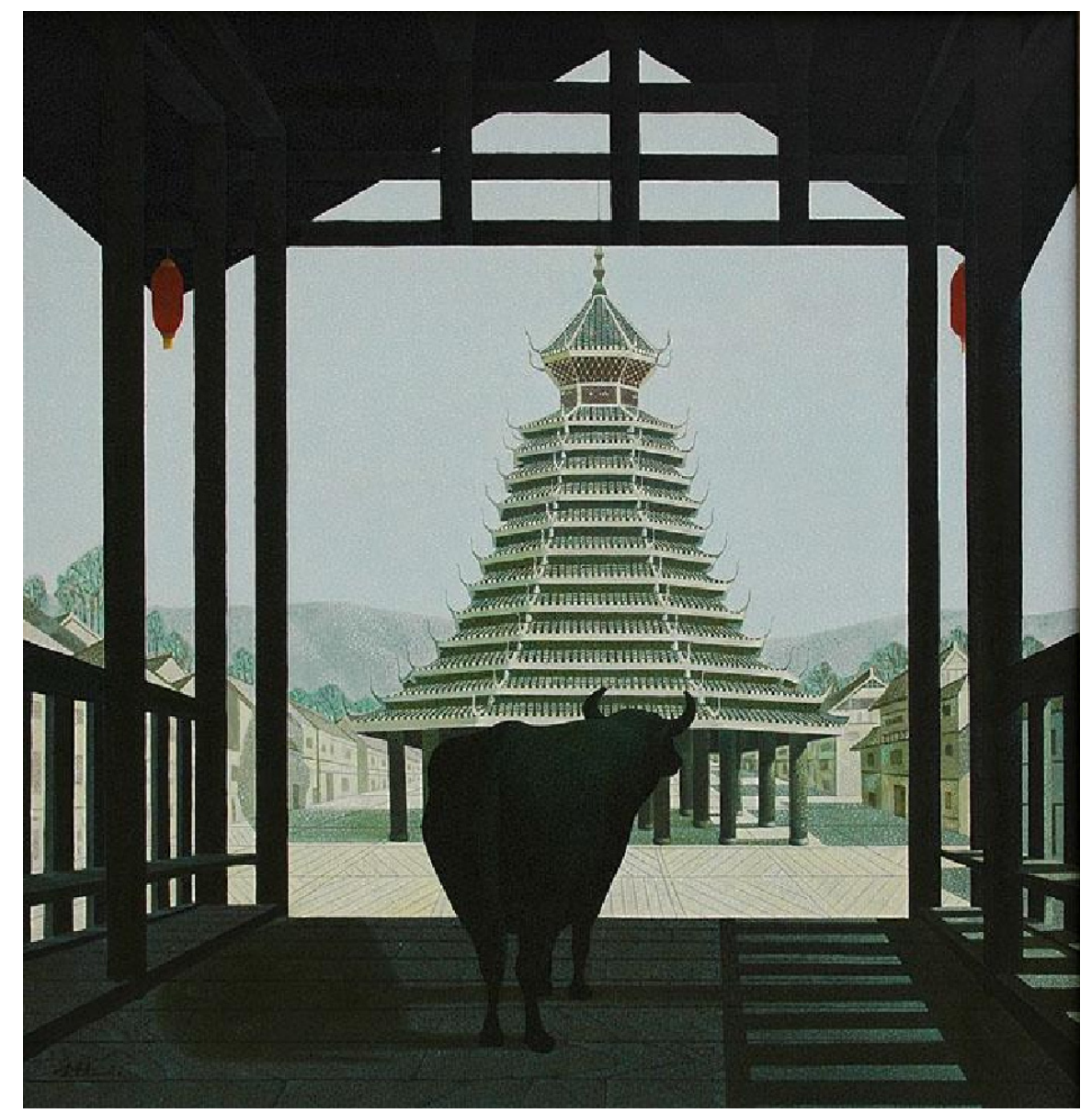

Figure 1. Passing Through the Wind and Rain Bridge (Li Huiyong 2006 Oil on canvas $100 \mathrm{~cm}$ * $100 \mathrm{~cm})$.

\subsection{Portrait of Characters}

There are many works depicting the portraits of ethnic minority figures, such as Wu Zuoren's Water Bearing Woman, Dong Xiwen's Kazakh Shepherdess, Zhan Jianjun's Song of the Plateau, Chen Danqing's Tibetan Painting and Jin Shangyi's Bride of Tajik. These popular oil paintings of portraits of ethnic minority have created the brilliance of Chinese oil painting of 20th century. In the face of Guizhou Miao village full of poetic and picturesque meanings and contains mysterious beauty, especially the typical Miao girls in Guizhou, their skin colour, face, eyes and spiritual temperament, together with beautiful headwear and gorgeous clothes, are shiny, golden, delicate, luxurious and real, which makes people admired. The main oil painters who create portraits of Miao people in Guizhou are Li Ang, Chen Xiaoguang and Zhaohong.

Chen Hongqi's oil painting Three Musketeers expresses vividly the rough, majestic and heroic temperament of the $\mathrm{Wu}$ Sha man. The most intuitive difference of the Wu Sha man is the unique hairstyle, which can best indicate them is sickle shave. Shaving with a sickle is a unique folk custom of the $\mathrm{Wu}$ Sha people, especially the skilful and sophisticated method of using a sickle by hairdressers, which is a legend of the $\mathrm{Wu}$ Sha people from generation to generation. The hair around the head is shaved with a sickle, but the top of the head is deliberately long hair pulled into a bun, hair tip naturally hanging, blown by wind, free and handsome. The man in $\mathrm{Wu}$ Sha wore a collarless lefthanded jacket, waist-stretched trousers, and oversized trousers, his legs tied up in the waist, a blue indigo and egg-white dyed bronze button jacket with a sloping placket, and a wide-legged trouser with a red belly, bright sunshine. The gun is a totem and symbol of Wu Sha. Every man has a gun of 
his own, a long gun and a short gun, a long gun on his shoulder, and a short gun on his waist. They have a special feeling with the gun, the gun does not leave people, and people do not leave the gun. Even after death, people also have to be buried with the gun (Figure 2).

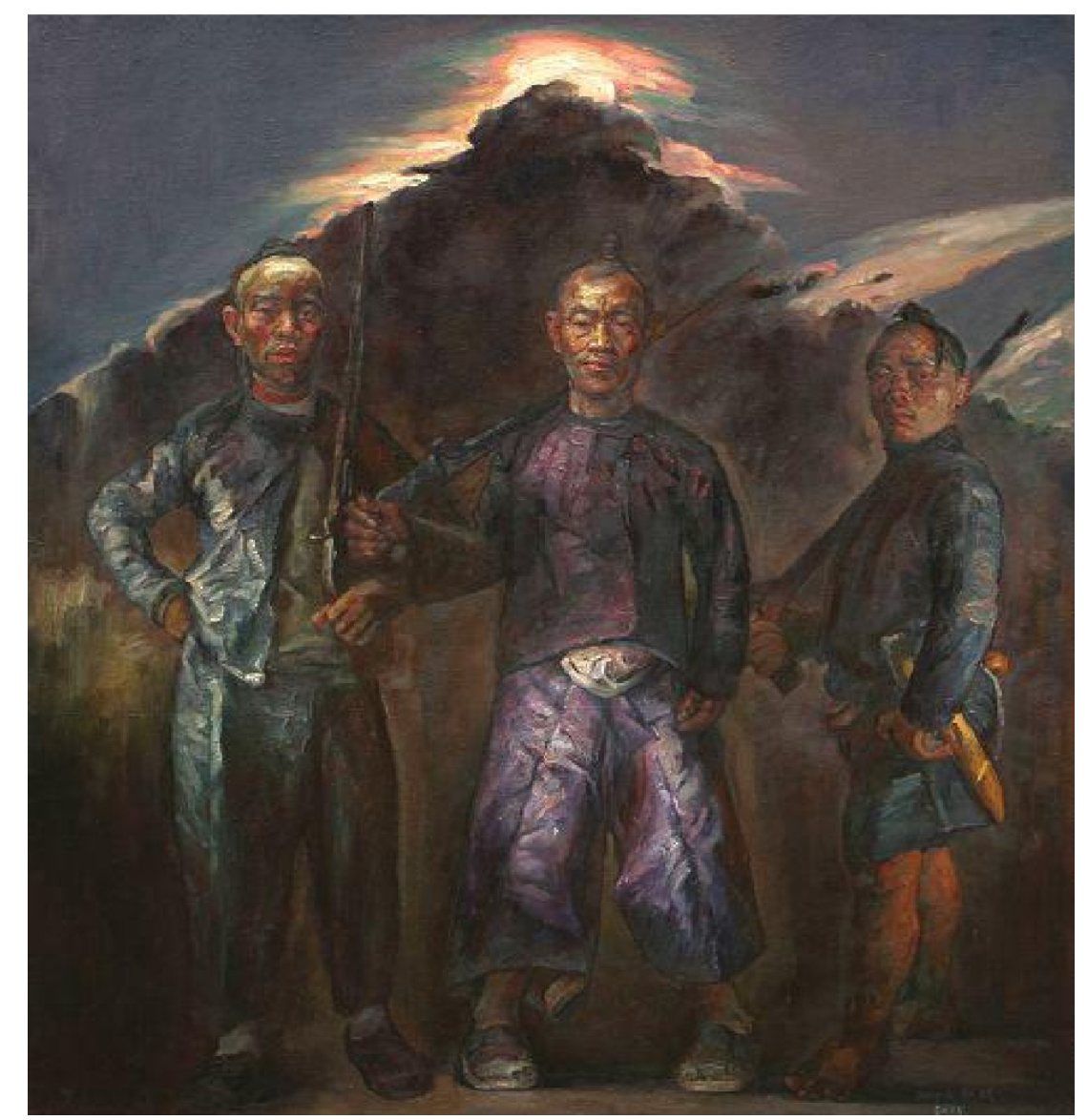

Figure 2. Three Musketeers (Chen Hongqi Oil on canvas, unknown size).

\subsection{Grand Festival Scene}

There are many festivals of Miao people in Guizhou, such as Lusheng Festival, Miaonian Festival, Kuaizang Festival and so on. Every festival, people will wear their own sewing clothes and wear some hand-made earrings, silver combs, silver hairpins and other dresses. Young men and women are good at singing and dancing. Love songs are lingering and pleasant, wine songs and blocking songs are bold and unrestrained, ancient songs are simple and heavy, each with its unique characteristics. With all these grand festivals, artists have gone all in to depict them.

Nowadays, many people disdain to show their real life in a realistic way. Zhao Jialing is obviously a guardian of traditional paintings. He has been working in this lonely land for decades. He has used rigorous and simple methods to portray the simple and honest image of these primitive econationalities and their lives of the Miao nationality. Each detail in the Miao nationality is the source of his artistic creation. For example, his work Wild Wu Sha with the theme of "Wu Sha Festival Carnival" as the subject matter depicts a group of such men with deep feelings and exquisite strokes. Those who stand in front of the paintings are all moved by the characters shown in the paintings. It is believed that painting is still a way to convince people with such power. Such a theme, exuding a strong soil fragrance and the joy of farmers, makes people feel the progress of the times and the modern atmosphere, and the nation's vigorous and wild of remote areas make people see the mystery behind the mountains and the continuation of national traditional culture. 


\subsection{Production and Living Scenario}

Every artist has his own unique artistic performance characteristics. It is possible to experience the ideological process that their inner heart experiences in the process of creation. For the production and living scenes of the Miao nationality in Guizhou that live in the original ecology and nature, everything is the artist's rare creative material.

Li Yuhui, Miao nationality, was born in 1965 in Yang Tun Village, Lei Shan County, Xijiang Town in Guizhou province. Most of his works mainly show the customs of Xijiang Miao village in south-eastern Guizhou, such as bullfight, drinking, market, farming, etc. They vividly reflect the production and life scenes of Xijiang Miao people. For example, his oil painting Rural Areas, the picture is the Miao people's rural business flourishing and lively extraordinary grand occasion, which is colourful. In Li Yuhui's paintings, slightly exaggerated characters and animals are short and strong, plain and humorous, clumsy and straightforward. Audiences not only feel the strong flavour of the Miao village in Xijiang, but also feel the painter's strong emotions towards his hometown.

\section{Conclusion}

Through full bloom and excellent results in oil paintings of Miao nationality in Guizhou, it can be said that an effective form of expression of the interpretation of ethnic minority culture is also the creative process to let viewers experience the charm of ethnic minority culture and art and artists' ideas. The oil paintings of Miao nationality in Guizhou are an important way for people to understand the Miao culture. As a student from Miao nationality in Guizhou, it is incumbent on us to devote ourselves to the artistic creation practice, and constantly absorb the essence of traditional culture, so as to integrate the traditional, local, national and times into western culture and to create an oil painting of his own personality style.

\section{References}

[1]. Chen, J. (2017). The influence of ethnic culture and social customs on the contemporary oil painting creation. Guizhou Ethnic Studies, 2, 400-406.

[2]. Gao, Y. H. (2016). On the teaching reform of curriculum of oil painting creation. Journal of Hubei Correspondence University, 3, 6-12.

[3]. Kong, H., Art, S. O., \& University, C. (2018). On the application of colour subjectivity in oil painting landscape creation. Journal of Bengbu University, 2, 106-112.

[4]. Zhang, Y., Cheng, J. (2016). Research on the expression of colour information in oil painting sketching creation. Journal of Zhengzhou University of Aeronautics, 6, 18-22.

[5]. Xia, R. (2017). The function of sketch in realistic oil painting. Agro Food Industry Hi Tech, 28(1), 3386-3388.

[6]. Wang, D. X. (2016). Chinese oil painting and Chinese image building in the new period. Journal of Kunming University, 1, 201-212. 